\title{
BRAF MUTATION ANALYSIS IN THYROID DISEASES- A STUDY FROM A TERTIARY CARE HOSPITAL/CENTRE IN KERALA
}

\author{
Priya P. Kartha' ${ }^{1}$ Santha Sadasivan ${ }^{2}$
}

${ }^{1}$ Assistant Professor, Department of Pathology, Integrated Institute of Medical Sciences, Palakkad.

${ }^{2}$ Professor, Department of Pathology, Sree Mookambika Institute of Medical Sciences, Kanyakumari, Tamilnadu.

\begin{abstract}
BACKGROUND

Thyroid nodules are present in approximately 5-10\% of adults on physical examination and in 50-70\% of people above 60 years of age on imaging, of which about 5-8\% of thyroid nodules are malignant. Proto-oncogenes that are particularly important in thyroid carcinogenesis include RET, TRK, and RAS. BRAF mutation is a major cause of aberrant activation of the MAP kinase pathway in human cancers. The T1799A (Thymine to adenine) mutation is the most common and virtually the only BRAF mutation identified in thyroid cancer and is associated with aggressive clinicopathological outcomes including tumour invasion, metastasis and recurrence.
\end{abstract}

Aim- To study the frequency of BRAF mutation in various thyroid diseases.

\section{MATERIALS AND METHODS}

A descriptive study which was done over a period of 2 years where all thyroidectomy specimens obtained during the study period were sampled. Histopathological study was done in Department of Pathology at our institute and molecular study done at Rajiv Gandhi Centre for Biotechnology, Thiruvananthapuram.

\section{RESULTS}

Most common lesion was multinodular goitre followed by solitary nodule. Papillary carcinoma accounted for $6.8 \%$ of cases. BRAF mutation was negative in all cases.

\section{CONCLUSION}

BRAF mutation is highly specific for papillary thyroid carcinoma with zero percent positivity in goitre, thyroiditis and other thyroid diseases. This suggests the possibility to evaluate thyroid nodules at molecular level preoperatively using FNAB to decide on further management.

\section{KEYWORDS}

Thyroid Lesions, Multinodular Goitre, BRAF, Papillary Carcinoma Thyroid, Molecular Study, PCR.

HOW TO CITE THIS ARTICLE: Kartha PP, Sadasivan S. BRAF mutation analysis in thyroid diseases- A study from a tertiary care hospital/centre in Kerala. J. Evolution Med. Dent. Sci. 2017;6(43):3359-3361, DOI: 10.14260/Jemds/2017/727

\section{BACKGROUND}

Thyroid nodules are present in approximately 5-10\% of adults on physical examination and in $50-70 \%$ of people above 60 years of age on imaging, of which about $5-8 \%$ of thyroid nodules are malignant.(1) A major effort in the clinical workup of a thyroid swelling is thus to determine whether it is a malignancy. ${ }^{(1,2)}$

The mitogen-activated protein kinase (MAPK) signalling pathway allows a cell to respond to external stimuli such as hormones and growth factors that interact with various receptors, including tyrosine kinase receptors like RET, and G protein coupled receptors like the TSH receptor.(2) Somatic genetic mutations are thought to cause the conversion of proto-oncogenes to oncogenes. Proto-oncogenes may or may not be expressed in normal cells, but there should be 2 copies

Financial or Other, Competing Interest: None.

Submission 21-04-2017, Peer Review 17-05-2017,

Acceptance 22-05-2017, Published 29-05-2017.

Corresponding Author:

Dr. Priya P. Kartha

Assistant Professor

Department of Pathology,

Integrated Institute of Medical Sciences,

Palakkad.

E-mail: priyapkartha@gmail.com

DOI: $10.14260 /$ jemds $/ 2017 / 727$

(c) $(1) \$$ of the wild-type gene in non-neoplastic tissues.(3) Many of these genes encode for growth factor receptors or for components of signal transduction pathways. When activating mutations occur, the overexpression of the oncogene product can result in uncontrolled growth and proliferation. Proto-oncogenes that are particularly important in thyroid carcinogenesis include RET, TRK, and RAS. $(2,3)$ RET/PTC rearrangement is a common activator of the MAP kinase pathway associated with thyroid malignancies.(4) RAS mutations, also can activate the MAP kinase pathway, which is found in thyroid cancers. One of the major causes of aberrant activation of the MAP kinase pathway is BRAF mutation. ${ }^{(5)}$

RAF kinases are of three types- A-RAF, B-RAF (BRAF), and C-RAF out of which BRAF is the most potent activator of the MAP kinase pathway. More than $90 \%$ of the mutations in BRAF gene is due to T1799A point mutation. This mutation causes a V600E amino acid change in the BRAF protein, which causes oncogenic activation of the BRAF kinase.(6) Discovery and characterisation of the T1799A BRAF mutation in thyroid cancer represent one of the most exciting advances in the molecular biology of thyroid cancer in recent years. In fact, this mutation is the most common known genetic alteration in thyroid cancer. A few other activated BRAF mutants are only rarely found in thyroid cancer. Numerous clinical studies demonstrated an association of BRAF 
mutation with aggressive clinicopathological outcomes, including tumour invasion, metastasis, and recurrence of Papillary carcinoma thyroid. $(6,7)$

The role of molecular markers in diagnosis and prognosis of thyroid cancers is promising.(8) The recent introduction of targeted therapeutic agents that have multiple targets, including the receptor tyrosine kinases, non-receptor tyrosine kinases, and serine-threonine kinases, has shown much promise in trials for patients with advanced thyroid cancer.(3) The current goal is to obtain a molecular profile of tumour so that it will be helpful in a treatment strategy with maximum response and minimum side effects.

Our attempt was to study the frequency of BRAF mutation in various thyroid diseases.

\section{MATERIALS AND METHODS}

Descriptive study done over a period of 2 years where all thyroidectomy specimens obtained during the study period were sampled. Morphological study done in Department of Pathology at our institute and molecular study done at Rajiv Gandhi Centre for Biotechnology, Thiruvananthapuram after ethical clearance. Those cases from which DNA and RNA could not be extracted were excluded.

Soon after thyroidectomy, tissue bits were taken from the suspicious areas on table and put in a solution called RNA LATER which helps in preventing degradation of RNA. The LATERS were then transported to Rajiv Gandhi Centre for Biotechnology where the specimens were stored at $-180^{\circ} \mathrm{C}$. The DNA isolated from the tissue biopsies by TRIzol method was amplified using PCR and then the amplified PCR product was used for sequencing PCR. The sequence of the samples, retrieved was aligned with the original mRNA sequence of BRAF to identify any mutation- i.e. Thymine to Adenine transition residing in the 1799 nucleotide position. Rest of the specimens were put in $10 \%$ formalin and sent to our department where gross and microscopic examination was done.

\section{RESULTS}

A total of 352 thyroidectomy cases were sampled. As per the exclusion criteria, 41 cases were excluded and finally a total of 311 cases were analysed in detail. Most common lesion was multinodular goitre (69\%), followed by solitary nodule (14.8\%). Next common lesion was diffuse thyromegaly which accounted for $5.5 \%$ of cases. Both non-neoplastic and neoplastic lesions were seen mainly in the age group $40-49$ years. Papillary carcinoma accounted for $6.8 \%$ of cases out of which $56 \%$ was classic papillary carcinoma, $22 \%$ papillary microcarcinoma, $18 \%$ follicular variant. Diffuse sclerosing and macrofollicular variants constituted $2 \%$ each. Other lesions seen were thyroiditis which constituted 2.3\%, hyperthyroidism and follicular neoplasms which made $1.6 \%$ of cases.

BRAF mutation analysis was done in all the 311 cases, and was negative in all as depicted in figure 1.

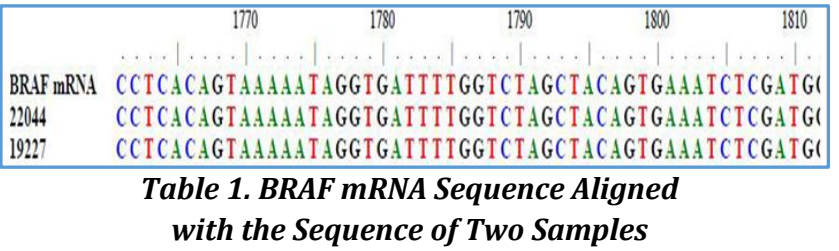

In this figure, the 1799 position corresponds to the region of mutation but no Thymine to Adenine transition was seen in any of the samples received.

\section{DISCUSSION}

According to Fugazzola, the positivity of BRAF mutation in papillary carcinoma thyroid ranges from 23 to $62 \%$ depending on the histological type, age group and epidemiological factors.(9) Most commonly seen associated with classic papillary type, BRAF positivity is strongly associated with poor prognostic factors like advanced age, lymph node metastasis and aggressive behaviour of the tumour.(9-13) According to Chakraborty A et al, the prevalence of BRAF mutation in papillary carcinoma thyroid in Indian cohort vary according to the subtype analysed, positivity being $61 \%$ in conventional papillary carcinoma.(14) A striking feature of BRAF mutation in thyroid malignancies is that it is exclusively seen in papillary thyroid carcinoma and papillary derived anaplastic thyroid carcinoma with a prevalence of $44 \%$ in the papillary carcinoma and $24 \%$ in the latter. It does not occur in follicular carcinoma or other thyroid lesions. $(6,10$, Although most of the studies proved a strong association between BRAF mutation and the high-risk clinicopathological features, there are studies that have failed to prove a significant association for which no definitive explanation could be given.(15-18) Comparable to the previous studies, BRAF mutation was negative in multinodular goitre and diffuse thyromegaly in our study, which proves that it is highly specific for papillary carcinoma. This highlights the significance of using BRAF mutational analysis in fine needle aspiration specimens so as to decide on further management and to avoid unnecessary thyroidectomies.

\section{Limitation}

Early detection of Papillary Carcinomas - most of our cases belong to age group less than 45 years. As BRAF mutation is usually associated with advanced age, it may be a cause for negativity.

Ours is the first study of this type in South India analysing the molecular level changes in thyroid lesions and we found that BRAF mutation being highly specific for papillary carcinoma thyroid. Hence, this study can be extended to aspiration cytology specimens for pre-operative diagnosis of the same. This avoids the need for unwanted surgeries. Further studies are also needed to analyse and type the BRAF mutations in papillary carcinoma thyroid and its variants as they are now goals for targeted therapy.

\section{CONCLUSION}

BRAF mutation was found to be highly specific for papillary carcinoma thyroid with zero percent positivity in nodular goitre, diffuse thyromegaly and other lesions. This emphasises the importance of carrying out BRAF mutation analysis in fine needle aspiration cytology specimens to detect papillary thyroid carcinomas and thus to decide on further management.

\section{REFERENCES}

[1] Mazzaferri EL. Management of a solitary thyroid nodule. N Engl J Med 1993;328(8):553-9. 
[2] Xing M. Diagnostic and prognostic molecular markers in thyroid cancer. In: Wartofsky L, Nostrand DV, (eds). Thyroid cancer. Springer New York 2016:281-9.

[3] Hunt JL. Molecular mutations in thyroid carcinogenesis. Am J Clin Pathol 2002;118 Suppl: S116-27.

[4] Ciampi R, Nikiforov YE. RET/PTC rearrangements and BRAF mutations in thyroid tumorigenesis. Endocrinology 2007;148(3):936-41.

[5] Davies H, Bignell GR, Cox C, et al. Mutations of the BRAF gene in human cancer. Nature 2002;417(6892):949-54.

[6] Xing M. BRAF mutation in thyroid cancer. Endocr Relat Cancer 2005;12(2):245-62.

[7] Garnett MJ, Marais R. Guilty as charged: B-RAF is a human oncogene. Cancer Cell 2004;6(4):313-9.

[8] Xing $M$, Haugen BR, Schlumberger M. Progress in molecular-based management of differentiated thyroid cancer. Lancet Lond Engl 2013;381(9871):1058-69.

[9] Fugazzola L, Puxeddu E, Avenia N, et al. Correlation between B-RafV600E mutation and clinico-pathologic parameters in papillary thyroid carcinoma: data from a multicentric Italian study and review of the literature. Endocr Relat Cancer 2006;13(2):455-64.

[10] Xing M. BRAF mutation in papillary thyroid cancer: pathogenic role, molecular bases, and clinical implications. Endocr Rev 2007;28(7):742-62.

[11] Adeniran AJ, Zhu Z, Gandhi M, et al. Correlation between genetic alterations and microscopic features, clinical manifestations, and prognostic characteristics of thyroid papillary carcinomas. Am J Surg Pathol 2006;30(2):216-22.
[12] Kebebew E, Weng J, Bauer J, et al. The prevalence and prognostic value of BRAF mutation in thyroid cancer. Ann Surg 2007;246(3):466-70.

[13] Nikiforova MN, Kimura ET, Gandhi M, et al. BRAF mutations in thyroid tumors are restricted to papillary carcinomas and anaplastic or poorly differentiated carcinomas arising from papillary carcinomas. J Clin Endocrinol Metab 2003;88(11):5399-404.

[14] Chakraborty A, Narkar A, Mukhopadhyaya R, et al. BRAF V600E mutation in papillary thyroid carcinoma: significant association with node metastases and extra thyroidal invasion. Endocr Pathol 2012;23(2):83-93.

[15] Liu C, Chen T, Liu Z. Associations between BRAFV600E and prognostic factors and poor outcomes in papillary thyroid carcinoma: a metaanalysis. World J Surg Oncol 2016;14(1):241.

[16] Fugazzola L, Mannavola D, Cirello V, et al. BRAF mutations in an Italian cohort of thyroid cancers. Clin Endocrinol 2004;61(2):239-43.

[17] Kim TY, Kim WB, Song JY, et al. The BRAF mutation is not associated with poor prognostic factors in Korean patients with conventional papillary thyroid microcarcinoma. Clin Endocrinol (Oxf) 2005;63(5):588-93.

[18] Liu RT, Chen YJ, Chou FF, et al. No correlation between BRAFV600E mutation and clinicopathological features of papillary thyroid carcinomas in Taiwan. Clin Endocrinol (Oxf) 2005;63(4):461-6. 